\title{
FATORES ASSOCIADOS À MORTALIDADE PRECOCE EM LISTA DE ESPERA DE TRANSPLANTE HEPÁTICO EM PACIENTES COM PONTUAÇÃO MELD BAIXA
}

\author{
Associated factors to the early death while on waiting list for liver transplantation \\ in patients with low MELD score
}

\begin{abstract}
Ivelise Regina Canito Brasil, José Huygens Parente Garcia, Cyntia Ferreira Gomes Viana, Tarciso Daniel Santos Rocha, João Batista Marinho Vasconcelos, Paulo Ewerton Garcia Costa, Gustavo Rego Coelho, Dirk Scherren, Fernanda Paula Cavalcante, Clébia Azevedo de Lima, Lêda Fátima Rocha Miranda, Flávia Amanso Campos, Marineuza Monteiro Silva Lucena
\end{abstract}

\section{RESUMO}

Introdução: 0 número de óbitos em lista de espera de transplante hepático é bastante elevado. No B rasil, foram realizados apenas 5,63 transplantes hepáticos/milhão de habitantes em 2005. Com a recente implantação no B rasil do critério de gravidade através do M ELD, espera-se que haja redução da mortalidade em fila de espera. Objetivo: A nalisar a relação entre os valores do M ELD e a presença de hiponatremia ou ascite ref ratária no momento da inclusão em lista, dos pacientes que apresentaram óbito precoce em lista de espera para transplante hepático. Método: A nál ise de 46 prontuários de pacientes que faleceram em até seis meses em lista de espera para transplante hepático. Foram estudados idade, sexo, classificação M EL D no momento da inclusão em lista, sódio sérico, presença de ascite refratária e tempo em lista até o óbito. Os pacientes foram divididos em dois grupos: grupo A com pacientes com M ELD inicial $\geq 16$ e grupo B com M ELD inicial <16. Os dois grupos foram comparados quanto a presença de ascite e/ou hiponatremia. Resultados: Tempo médio de três meses até 0 óbito. 0 total de pacientes nos grupos A e B foi respectivamente de 31 e 15. No grupo B, 93\% dos pacientes apresentavam hiponatremia e/ou ascite. Não houve diferença estatística quanto a presença de hiponatremia e/ou ascite ref ratária entre grupos. Condusão. A maioria dos pacientes com M ELD <16 que evoluíram para óbito precoce em lista apresentou hiponatremia e/ou ascite refratária.

Descritores: Transplante de Fígado; Listas de Espera; A locação de Recursos/ M étodos; Hiponatremia; A scite.

\section{Instituição:}

Centro de Transplante de Fígado do Hospital Universitário Walter Cantidio da Universidade Federal do Ceará

\section{Correspondência:}

Dra. Ivelise Regina Canito Brasil

Centro de Transplante de Fígado do Ceará

Rua Cap. Francisco Pedro, 1290 - Rodolfo Teófilo

CEP 60.430-370 - Fortaleza - CE - Brasil

Fone: (85) 3366.8235 / Fax: (85) 3366.8581

E-mail: ivesbrasil@hotmail.com

Recebido em: 01.03.2006

Aceito em: 25.05.2006

\section{INTRODUÇÃO}

0 primeiro transplante de fígado no estado do Ceará foi realizado em 18 de maio de 2002. Desde então, até janei ro de 2006, o serviço de transplante de fígado do Hospital das Clínicas da Universidade Federal do Ceará realizou 154 transplantes com doador falecido, beneficiando um total de 143 pacientes listados primariamente no estado ou oriundos dos diversos serviços credenciados pelo Sistema Nacional de Transplantes (SN T) do B rasil. A tualmente, existem 166 pacientes em lista de espera por um órgão no C eará. A pesar da real ização de um número significativo de transplantes - mais de 50 transplantes/ano - houve nos últimos dois anos 101 óbitos na lista de espera (Tabela 1).

Tabela 1. Distribuição dos Pacientes do Centro de Transplante de Fígado do Ceará de maio/2002 a janeiro/2006

\begin{tabular}{cc} 
PACIENTES & Valor/porcentagem \\
\hline ÓBITOS EM LISTA & $101(23,45 \%)$ \\
TRANSPLANTADOS & $146(35,4 \%)$ \\
ATUALMENTE EM LISTA & $166(40,1 \%)$ \\
\hline TOTAL DE PACIENTES & $\mathbf{4 1 3}(\mathbf{1 0 0} \%)$
\end{tabular}


Segundo o IBGE, a população do estado do Ceará é de aproximadamente 7.430 .611 habitantes. ${ }^{1} \mathrm{C}$ onsiderando-se os estados que não possuem serviço de transplante hepático (R io G rande do N orte, M aranhão e Piauí, além de toda a R egião N orte), a população total coberta pelo serviço do estado do Ceará aproxima-se de 30 milhões de habitantes. A necessidade estimada de transplante de fígado apenas para pacientes oriundos do Ceará é de 150 por ano, havendo, portanto, um déficit de 100 transplantes/ano. Esses pacientes estão provavel mente morrendo em lista de espera ou não têm acesso a um serviço especializado.

0 número de óbitos em lista de espera em todo o mundo é bastante elevado, e este fato é ainda mais grave no Brasil. Em 2005 foram realizados apenas 5,63 transpl antes hepáticos/milhão de habitantes neste país, o que está muito abaixo da necessidade nacional. ${ }^{2}$

No momento de inclusão em lista, o M ELD (M odel for End-stage Liver Disease) tem se mostrado capaz de prever a mortalidade em três meses para adultos. ${ }^{3} 0$ sistema de alocação de órgãos por gravidade baseado na escala M ELD beneficia pacientes com doença hepática mais avançada, e, provavelmente, diminui a mortalidade em lista de espera. Os EUA adotaram o sistema M EL D em fevereiro de 2002, sendo seguido por outros países. Um estudo realizado na M ayo Clinic, em pacientes com doença hepática crônica, mostrou que 412 (12\%) pacientes morreram durante os três meses iniciais de seguimento, e que esta mortalidade foi diretamente proporcional ao MELD inicial. Pacientes com MELD inicial inferior a nove apresentaram mortal idade de $1,9 \%$ nos três primeiros meses, enquanto aqueles com M ELD inicial maior ou igual a 40 apresentaram uma taxa de mortal idade de $71,3 \% .{ }^{4}$

A presença de ascite refratária, um conhecido preditor de mortalidade em lista, não é abordada pelo sistema M ELD. Esta condição, que está associada a níveis baixos de sódio sérico, tem sido considerada, em alguns serviços, um fator independente de mortal idade em lista. A associação entre sódio sérico inferior a 126 $\mathrm{mEq} / \mathrm{dl}$ e M ELD parece ser superior ao M ELD isolado em prever a mortalidade nos primeiros três a seis meses de lista de espera. ${ }^{5}$

O B rasil adotava o critério cronológico para alocação de fígados. Porém, com a recente implantação do critério de gravidade, estimase que haja redução da mortal idade em fila de espera.

0 objetivo deste trabalho é analisar a relação entre o perfil da escala M ELD e a presença de hiponatremia ou de ascite intratável no momento da inclusão em lista em pacientes que apresentaram óbito precoce em lista de espera para transplante hepático no estado do Ceará.

\section{MÉTODOS}

Foi realizada análise retrospectiva de 46 prontuários de pacientes que faleceram precocemente em lista de espera para transplante hepático, entre maio de 2002 e janeiro de 2006. Foram excluídos pacientes com doença colestática, hepatite fulminante e aqueles com dados incompletos no prontuário. A nal isaram-se idade, sexo, classificação M ELD no momento da inclusão em lista, sódio sérico, presença de ascite refratária e tempo em lista até o óbito de todos os pacientes. Os pacientes foram divididos em dois grupos: $G$ rupo A com pacientes com M ELD inicial igual ou superior a 16; Grupo $B$ aqueles com M ELD inicial menor que 16 . Considerou-se óbito precoce aquele ocorrido nos primei ros seis meses de lista de espera. A scite refratária foi definida como aquela não responsiva a $400 \mathrm{mg}$ de espironolactona associada a até $120 \mathrm{mg}$ de furosemida diária durante duas semanas.

\section{RESULTADOS}

Um total de 46 pacientes, $34(73,9 \%)$ homens e $12(26,1 \%)$ mulheres, foram estudados. A média de idade por ocasião do óbito foi de 48,4 anos (12-65). 0 tempo médio entre a inscrição e o óbito foi de três meses (0-6).

O grupo A foi formado por $31(67,4 \%)$ pacientes e o grupo B por $15(32,6 \%)$ pacientes. A scite refratária foi diagnosticada em sete $(22,6 \%)$ pacientes no grupo A e em sete $(46,7 \%)$ no grupo B. Em relação à hiponatremia, os valores foram $15(48,4 \%)$ e sete $(46,7 \%)$, nos grupos A e B respectivamente. Avaliando-se a presença de ascite e/ou hiponatremia, no grupo A 22 (71\%) e no grupo B $14(93 \%)$, os pacientes apresentavam pelo menos uma das duas variáveis. N enhuma das diferenças entre os grupos apresentou-se como estatisticamente significante $(p>0,05)$. A Tabela 2 resume as principais variáveis analisadas no estudo.

Tabela 2 - Perfil dos pacientes que foram a óbito precocemente em lista de espera para Transplante Hepático no estado do Ceará.

\begin{tabular}{cc}
\hline PERFIL DOS PACIENTES & Valor / variação \\
\hline Total de Pacientes & 46 \\
Idade Média ao Óbito (anos) & $48,4(12-65)$ \\
Masculino/Feminino & $34(73,9 \%) / 12(26,1 \%)$ \\
Tempo médio entre a inscrição & $3(0-6)$ \\
e o óbito (meses) & $14(30,43 \%)$ \\
Presença de ascite refratária & $31(67,4 \%)$ \\
MELD $\geq 16$ & $22(47,82 \%)$ \\
Sódio sérico < 135
\end{tabular}

\section{DISCUSSÃO}

A elevada mortalidade de pacientes em lista de espera para transplante hepático é, ai nda hoje, uma real idade. No estado do C eará, em um período de 43 meses, 154 transplantes de fígado foram realizados. Entretanto, no mesmo período, 101 pacientes foram a óbito em lista de espera.

0 principal fator limitante do número de transplantes hepáticos realizados no Brasil é, sem dúvida, a escassez de doadores. U ma política nacional efetiva de estímulo à doação de órgãos poderia aumentar consideravel mente o número de doadores e, assim, contribuir adicional mente para a redução da mortalidade de pacientes em lista de espera.

Têm sido propostas al ternativas para diminuir a mortal idade em lista, como a adoção do critério de gravidade através do sistema M ELD, que acaba de ser implementado no B rasil. 0 transplante com doadores vivos também tem sido utilizado como alternativa à falta de doadores falecidos. 6

Nesta realidade de escassez de doadores, a adoção do sistema de gravidade através do M ELD talvez seja capaz de diminuir a mortalidade em lista. Entretanto, alguns autores analisaram quatro sistemas de gravidade (C hild, M ELD, F reeman Scale e Guardiola) e concluíram que nenhum del es era capaz de prever 
com acurácia o prognóstico dos pacientes em lista de espera. ${ }^{7}$ A Iguns estudos consideram o nível sódio sérico menor que 135 $\mathrm{mEq} / \mathrm{dl}$ um fator independente de mortalidade precoce em lista. ${ }^{8}$ Esses estudos sugerem que a adição do nível de sódio sérico ao MELD poderia aumentar o valor preditivo de mortalidade em lista de espera. ${ }^{5}$

Neste estudo, a maioria dos pacientes que evoluíram para óbito precoce apresentavam MELD inicial $\geq 16$. Entretanto, uma parcela significativa de $32,6 \%$ dos pacientes apresentava M EL D menor que 16, e evoluiu precocemente para óbito.

Esses dados revelaram que em mais de $30 \%$ dos casos, o M ELD inicial não apontou para um risco de mortalidade precoce, porém ascite e/ou hiponatremia estavam presentes em 93\% destes pacientes.

E mbora os dados apresentados tenham importância clínica, não foi encontrada diferença estatisticamente significante entre os grupos $\mathrm{A}$ e $\mathrm{B}$ em relação à presença de hiponatremia e/ou ascite refratária. Contudo, em uma casuística maior, é possível que essa diferença venha a se tornar significativa.

\section{CONCLUSÃO}

Neste estudo, a maioria dos pacientes com M ELD $<16$, que evoluíram para óbito precoce em lista, apresentava hi ponatremia e/ou ascite refratária.

\begin{abstract}
Introduction: M ortality on waiting list for liver transplantation is high around the world. In B razil, 5.63 liver transplantation/million people were performed along 2005. With the recent implantation in B razil of the severity according to the M EL D score, there might be a reduction in the amount of death on waiting list. Purpose: This paper will analize the relationship between M EL D score and hyponatremia or refractory ascites upon the registration on waiting list for liver transplantation of patients who died in the first six months of list. Patients and Methods: A nalysis of 46 records of patients who died in the first 6 months on waiting list for liver transplantation was performed. It was assessed the age, gender, initial M ELD score, serum sodium, refractory ascites, and time on the list until the death. Patients were divided in 2 groups: group A, patients with initial MELD score $\geq 16$, and group B those with initial M ELD score $<16$. $B$ oth groups were compared as to the presence of refractory ascites and/or hyponatremia. Results: M ean time until death was 3 months. Total amount of patients in group A and B was 31 and 15 respectively. In group B, 93\% patients showed either hyponatremia or ascites. There was no statistically significant difference as to the presence of hyponatremia and/or ascites between both groups. Conclusion: M ost patients with M ELD $<16$ who died in the first 6 moths on the waiting list had hyponatremia and/or refractory ascites.
\end{abstract}

Keywords: Liver Transplantation, Waiting Lists, Organ A llocation, Hyponatremia, A scites.

\section{REFERÊNCIAS}

1- Instituto Brasileiro de Geografia e Estatística(IBGE): Estados. Disponível em http:// www.ibge.gov.br/estadosat/perfil.php?sigla=ce.

2- Associação Brasileira de Transplante de Órgãos (ABTO). Disponível em http://www. abto.org.br/profissionais/profissionais.asp\#.

3- Watt KD, Menke T, Lyden E, McCashland TM. Mortality while awaiting liver retransplantation: predictability of MELD scores. Liver Transpl. 2005;37(5):2172-3.

4- Wiesner R, Edwards E, Freeman R, Harper A, Kim R, Kamath P, et al. Model for end-stage liver disease (MELD) and allocation of donor livers. Gastroenterology. 2003;124(1):91-6.
5- Biggins SW, Rodriguez HJ, Bacchetti P, Bass NM, Roberts JP, Terrault NA. Serum sodium predicts mortality in patients listed for liver transplantation. Hepatology. 2005;41(1):32-9.

6- Russo MW, LaPointe-Rudow D, Kinkhabwala M, Emond J, Brown RS Jr. Impact of adult living donor liver transplantation on waiting time survival in candidates listed for liver transplantation. Am J Transpl. 2004;4(3):427-31.

7- $\quad$ Llado L, Figueras J, Memba R, Xiol X, Baliellas C, Vazquez S, et al. Is MELD really the definitive score for liver allocation? Liver Transpl. 2002;8(9):795-8.

8- Heuman DM, Abou-Assi SG, Habib A, Williams LM, Stravitz RT, Sanyal AJ, et al. Persistent ascites and low serum sodium identify patients with cirrhosis and low MELD scores who are at high risk for early death. Hepatology. 2004;40(4):802-10. 